\title{
Macrólidos en enfermedades inflamatorias crónicas de las vías respiratorias
}

\section{Macrolides in chronic inflammatory diseases of the airway}

Mauricio Moreno(1); Oscar Sáenz Morales ${ }^{(2)}$; Camilo Manrique Martínez ${ }^{(3) ;}$ Francisco González Acosta( ${ }^{(4)}$; Erika Paola Vergara Vela(5); Alberto Mario Pereira Garzón ${ }^{(6)}$; Rafael Miranda Jiménez ${ }^{(7)}$

\section{RESUMEN}

Revisamos la actividad de los macrólidos en la enfermedad inflamatoria crónica de la vía aérea, haciendo énfasis en los efectos inmunomoduladores y antiinflamatorios sobre la célula epitelial. Describimos en detalle los mediadores humorales, celulares y las diferentes interacciones entre estos antibióticos y los mediadores inflamatorios circulantes, incluyendo procesos de apoptosis y oxidación, así como efectos sobre la defensa del epitelio, acciones sobre el moco, movimiento de iones, defensinas, depuración mucociliar y finalmente estabilización epitelial. Debido a estos mecanismos descritos, los macrólidos se convierten en una alternativa coadyuvante en el manejo de las enfermedades pulmonares infecciosas e inflamatorias.

Palabras clave: macrólidos, efectos inmunomodulatorios, célula epitelial, enfermedad inflamatoria de la vía aérea, mediadores inflamatorios.

\section{ABSTRACT}

We review the activity of macrolides in chronic inflammatory airway disease, laying emphasis on their immunomodulatory and anti-inflammatory effects on the epithelial cell. We describe in detail the humoral and cellular mediators involved, as well as the interactions between these antibiotics and circulating inflammatory mediators (including processes of apoptosis and oxidation), along with the effects on epithelial defenses, actions on mucus, movement of ions, defensins, mucociliary clearance, and epithelial stabilization. Because of these mechanisms, macrolides have become an adjuvant in the management of infectious and inflammatory lung diseases.

Keywords: macrolides, immunomodulatory effects, epithelial cell, inflammatory airway disease, inflammatory mediators.

Rev Colomb Neumol 2011; 23(2): 40-47.

\section{INTRODUCCIÓN}

La enfermedad pulmonar obstructiva crónica (EPOC) constituye un problema de salud pública de gran importancia; tiene característicamente un curso crónico y progresivo con exacerbaciones frecuentes que puede conducir incapacidad y muerte. Representa una causa frecuente de morbi mortalidad en nuestro país, (1). Es una entidad que empieza a hacer predominante a partir de la quinta década de la vida, especialmente en varones, afectando principalmente a personas mayores de 65 años $(2,3)$.

(1) Medicina Interna Hospital Santa Clara.mamg256@gmail.com

(2) Internista Neumólogo. Coordinador servicio urgencias Hospital Santa Clara. Coordinador servicio medicina interna Saludcoop EPS, docente facultad de Medicina Universidad el Bosque. oasm15@yahoo.com

(3) Medico Internista. Coordinador Servicio urgencias Hospital Santa Clara. Docente facultad de Medicina Universidad el Bosque. camiloandresmanrique@gmail.com

(4) Farmacólogo. Bioeticista. Servicio urgencias Hospital Santa Clara. Docente facultad de Medicina Universidad el Bosque. gonzalezfrancisco@unbosque.edu.co

(5) Medico Internista. Servicio urgencias Hospital Santa Clara.paoli1982@gmail.com

(6) Medico Internista. Servicio urgencias Hospital Santa Clara. rolopererira12hotmail.com

(7) Medico Epidemiólogo. Servicio de urgencias Hospital Santa Clara. rafaelmirandaj@gmail.com

Correspondencia: Doctor Mauricio Moreno. Correo electrónico: mamg256@gmail.com

Recibido: 28 de Julio de 2011. Aceptado: 12 de Agosto de 2011. 
Estos precedentes, además de darnos una idea de la magnitud del problema, justifican en sí mismos la iniciativa de nuestras sociedades científicas de realizar una puesta en común, desde diversos puntos de vista, de las peculiaridades y de la problemática en la atención del paciente con EPOC. Importantes avances terapéuticos se han tenido en los últimos 20 años llamando la atención de los investigadores el papel de algunos agentes antibióticos, no por su espectro bactericida, si no por su efecto inmunomodulador, por lo anterior agentes como sulfonamidas, lincosamidas, tetraciclinas, amino glucósidos, fluoroquinolonas, betaláctamicos, y finalmente macrólidos

El daño del epitelio bronquial y la hipersecreción mucosa son características de la inflamación crónica de las vías respiratorias que pueden afectar la depuración mucociliar y pueden causar recurrentes o persistentes episodios de infecciones respiratorias. En respuesta a factores quimiotaticos producidos por los tejidos dañados o inflamados, los neutrófilos se mueven a través de etapas sucesivas de reclutamiento y se constituyen en parte fundamental en la actividad bactericida por fagocitosis, liberación de enzimas lisosomales, y la generación de especies reactivas de oxígeno, síntesis y liberación de citoquinas pro inflamatorias. Los datos confirman que los antibióticos macrólidos tienen propiedades clasificadas o denominadas como no bactericidas que incluyen 1. Inhibición de quimiotaxis de células inflamatorias y citoquinas, 2 . Inhibición de la expresión de la síntesis de moléculas de adhesión, 3. Producción de especies reactivas de oxígeno, 4. Disminución de la hipersecreción de moco de las vías respiratorias en pacientes con panbronquiolitis difusa crónica, la sinusitis y la bronquitis crónica.

Los macrólidos se acumulan en los neutrófilos y macrófagos en concentraciones significativamente más altas que en el líquido extracelular. (52)

En esta revisión nos dedicaremos al papel de los macrolidos, y su importancia en el tratamiento de patologías como panbronquiolitis difusa, fibrosis quística, asma y principalmente su papel en enfermedad pulmonar obstructiva crónica.

Iniciamos este interesante tema definiendo a los macrólidos como agentes bacterio estáticos que inician su presentación en 1952 con el surgimiento de la eritromicina, son una clase de moléculas con un anillo sustituido de lactonamacrocíclica, es una familia bien caracterizada de antimicrobianos de amplio espectro, que inhiben la síntesis de proteínas bacterianas a través de interacciones con el RNA ribosomal $23 S$ ribosomal y proteínas específicas. Macrólidos, incluyendo los 14 miembros de la eritromicina, roxitromicina y claritromicina y azitromicina 15-miembros, son activos frente a gram positivos y negativos de las bacterias y también modula la producción de factores de virulencia de bacterias y toxinas (4). Recientemente, su capacidad para modular las funciones celulares y actuar como inmunomoduladores ha comenzado a ser plenamente conocida (5). La acumulación de pruebas ahora soporta el uso de estos fármacos como terapia inmunomoduladoraen el tratamiento de enfermedades pulmonares crónicas, como fibrosis quística, asma y la EPOC (6-11).

Es importante definir los mecanismos o efectos no antibióticos de los macrólidos, los cuales podemos agrupar en 8 tópicos, dentro de los cuales están :

- Macrólidos como medicamentos antiinflamatorios o inmunomoduladores

- $\quad$ Acción sobre los neutrófilos

- Efecto sobre la defensa del epitelio

- $\quad$ Efecto sobre el moco

- Efecto sobre el movimiento de iones

- $\quad$ Efecto sobre las defensinas

- Efecto sobre la barrera de la estabilidad del epitelio.

A pesar de estar demostrado en estudios in vitro el efecto inmunomodulador de los macrolidos debemos aclarar que faltan estudios aleatorizados y controlados que permitan establecer el uso de estos medicamentos en nuestra práctica cotidiana, teniendo presente el riesgo de inducir resistencia antibiótica. Por lo anterior la invitación es a la buena práctica fundamentada en estudios los cuales deberán ser realizados en los próximos años, para determinar si nuestro hallazgo en laboratorio pudiera ser extrapolable en nuestra práctica clínica.

\section{ABREVIATURAS:}

- IL-8INTEREUQUINA 8

- TNF FACTOR DE NECROSIS TUMORAL

- LPS LIPOPOLISACARIDOS

- ICAM-1 MOLECULAS DE ADHESION INTERCELULAR

- BET-1A LINEADE CELULAS EPITELIALES ... BRONQUIALES

- INF INTERFERON

- IB3-1 CULTIVO DE CEL EPITELIALES NASALES

- CFTR GEN REGULADOR DE CONDUCTANCIADE TRANSMEMBRANA 
- NFêB FACTOR NUCLEAR DE CADENA KAPPA ESTIMULANTE CEL B

- AP-1 FACTOR TRANSCRIPCIONALDE APOPRETINA 1

- BET línea 1AINTERFERON BETAA1

- GM-CSF FACTOR ESTIMULANTE DE COLONIAS GRANULOCITICOMONOCITICO

- MUC5AC GEN DE MUCOPROTEINA

- ERK PROTEINAS DE FAMILIA KINASAS

- MUC2 PROTEINAS OLIGOMERICAS MOCO

- CFTR REGULADOR DE CONDUCTANCIADE TRANSMEMBRANA

- ENAC CANAL EPITELIAL DE SODIO

- BEAS-2B CELULAS EPITELIALES BRONQUIALES HUMANAS

- A549LINEACELULAR EPITELIALALVEOLAR HUMANA

\section{FUNCIONES DE LOS NEUTRÓFILOS}

Los neutrófilos actúan de forma selectiva, con un comportamiento pro inflamatorio es decir integran múltiples señales derivadas de la alta afinidad de las integrinas, citoquinas pro inflamatorias, y de quimio atrayentes producida por las bacterias y las células epiteliales las cuales surgen en respuesta a las noxas en los diferentes tejidos. Históricamente, los neutrófilos han sido considerados carentes de actividad transcripcional. Sin embargo, la evidencia molecular indica ahora que los neutrófilos humanos son una fuente de diversos agentes proinflamatorios citocinas, quimiocinas y factores de crecimiento.

Los tipos de estímulos, incluidas las enfermedades, endotoxinas, citoquinas reguladoras, y quimiocinas, influye en el patrón de citoquinas pro-neutrofílica a producir. Se sabe que la IL-8 expresada en respuesta al lipopolisacárido (LPS), tumor necrosis factor (TNF), además de inmunocomplejos son respuestas mediadas por los neutrófilos. Una variedad de células, incluyendo los neutrófilos, Tlinfocitos y las células epiteliales, secretan IL8, que a su vez estimula el reclutamiento y activación deneutrófilos. Así, los neutrófilos son el principal origen y el destino celular primaria de IL8. Además de las citocinas, los neutrófilos son reclutados para liberar enzimas en la mucosa de las vías respiratorias y super aniones óxido. Las proteasas neutrofilicas y sus derivados, incluyendo el astasa y la proteinasa 3 , estimulan la degracion de los gránulos de mucina y la liberación de glicoproteína por las células caliciformes y glándulas submucosas.

\section{MACROLIDOS Y RESPUESTA INMUNOMODULADORA:}

El sistema inmunológico es un sistema complejo, dinámico y redundante con interacciones (9). La modulación de este sistema es un ciclo no lineal de la activación y el ciclo de la supresión de alrededor de un agente homeostático. Inmunomodulación es, pues, un concepto diferente de la inmunosupresión. La respuesta a dosis bajas de los agentes macrolidos, ha mostrado un efecto inmunomodulador y se ha determinado que cuando se dosifica para efecto antibiótico este efecto inmunomodulador no se presenta (12-14). Como ejemplos de la terapia de dosis bajas, la eficacia a largo plazo y la seguridad de claritromicina de 200 mg/día se evaluó en 10 pacientes con Panbronquiolitis Difusa (DPB) que fueron tratados durante 4 años. La función pulmonar mejoró en la mayoría de los pacientes dentro de los 6 meses de iniciar el tratamiento, la terapia fue bien tolerada (14 ). En la Fibrosis Quistica (FQ), Saiman et al. Ilevó a cabo un gran estudio multicéntrico, aleatorizado y controlado, con 185 sujetos de 6 años o mayores, que estaban crónicamente infectados con Pseudomonasaeruginosa. Los sujetos fueron asignados aleatoriamente a recibir $250 \mathrm{mg}$ (peso $<40 \mathrm{~kg}$ ) o $500 \mathrm{mg}$ (peso > o $=40 \mathrm{~kg}$ ) de azitromicina por vía oral 3 días por semana durante 6 meses. Después de 6 meses el grupo tratado con azitromicina presentaron un incremento medio del 6,21\% para FEV1 y FVC de 4,95\% en comparación con el grupo placebo, hubo menos exacerbaciones que en el grupo placebo (15).

Las propiedades inmunomoduladoras de los macrólidos están bien documentados, tanto in vitro como in vivo (7), (15-17). Los mecanismos propuestos para estos efectos inmunomoduladores han sido recientemente revisado por Shinkai et al. (18), y por Parnham y colegas (7), (15), (19) y (20). Es de destacar que la capacidad de los macrólidos a acumularse en las células del huésped, incluyendo los fagocitos y las células epiteliales, pueden desempeñar un papel en la regulación de las funciones celulares de estas moléculas. La acumulación en las células del huésped puede variar dependiendo del tipo de célula macrólidos y considera (21), en consonancia con los efectos inmunomoduladores, trabajos recientes sugieren que el 14 - y 15-macrólidos miembro puede facilitar la resolución de la inflamación. Claritromicina y azitromicina puede aumentar la fagocitosis de las células epiteliales de apoptosis (22) y neutrófilos (23) por los macrófagos. Esta función de liquidación fagocítica se ve afectada en la EPOC y agrava la inflamación crónica de esta enfermedad $(24,25)$. En consecuencia con estos efectos, se sugiere un mecanismo de regulación transcripcional, el cual inhibe la síntesis de colágeno tipo I en los fibroblastos dérmicos 
(26). Estos informes muestran que algunos de estos nuevos efectos de los macrólidos son independientes de su actividad antimicrobiana.

los cultivos de células epiteliales en una operación de concentración-dependiente entre 1 y $25 \mathrm{~m} \mathrm{~g} / \mathrm{mL}$. Los resultados de este estudio sugieren que roxitromicina puede disminuir la número de neutrófilos en líquido del lavado broncoalveolar de pacientes con la inflamación de las vías respiratorias en un proceso que difiere de su actividad antibiótica. Kawasaki y colleagues (55) emplearon una enzima de la célula ligada por un método inmunoenzimático de ensayo para evaluar el papel de ICAM-1 en el reclutamiento de neutrófilos en las vías respiratorias. El ser humano tiene una línea de células epiteliales bronquiales (Bet-1A), se cultivan y son tratados con IFN- con o sin roxitromicina por 18 horas, seguido por la adición de anticuerpos ICAM-1 con anticuerpos humanos monoclonales. IFN aumenta la expresión de ICAM-1 en la célula epitelial superficies, pero roxitromicina disminuyó significativamente de la magnitud de su expresión, de nuevo en una concentración de forma dependiente. Estos hallazgos sugieren que los derivados de la eritromicina $A$ (en este caso, roxitromicina) modula indirectamente el reclutamiento de los neutrófilos

Figura 1. Tomada y Adaptada de Chest 2004. The Effects of Macrolides on inflammatory cells, páginas 41S-51S.

\section{MACROLIDOS Y EFECTOS EN EL NEUTROFILO}

Hay cierto desacuerdo en cuanto a los efectos de la antibióticos macrólidos sobre la migración de los neutrófilos. Hasta la fecha, los efectos inhibitorios en vitro y en vivo (53-54) se han descrito, mientras que otros han informado de ningún efecto en vitro o efectos estimulantes en el in vitro y en vivo. (54) La razón de esta controversia es desconocido, pero podría deberse a diferencias en las especies, y/o las condiciones experimentales. Kawasaki et al (55) llevó a cabo un estudio in vitro de los efectos potenciales de roxitromicina sobre la adhesión de los neutrófilos de bronquios las células epiteliales y el papel de ICAM-1. El pretratamiento de los neutrófilos con la N-formil-metionina - leucilfenilalanina, pero no con LPS, de manera significativa producía una mayor adherencia. La estimulación de bronquios humanos y las células epiteliales con interferón (IFN) y TNF También aumento la adhesión de los neutrófilos regulado por las células epiteliales con la mediación de la interleuquina 8. Cuando los neutrófilos pretratados con $\mathrm{N}$-formilmetionina-leucil-fenil - alanina, se exponen a roxitromicina esta inhibe la adhesión de los neutrófilos a a los sitios inflamados por la supresión de la de expresión de ICAM-1. Estudios de Dr Francis Couturaud et al ha demostrado que el LPS aumenta la expresión de ICAM-1 cuando se añade al cultivo células epiteliales traqueales en murinos, y que la preincubación de las células con claritromicina redujo significativamen-te expresión de ICAM-1 en una concentración-dependiente. En contraste, las concentraciones altas de amoxicilina, cefaclor, o amikacina no demostró en este sentido (K. Isuno, J. Tamaoki, M. Kondo, A. Nagai, la observación inédita; de mayo 2001), sugiriendo que el efecto podría ser específico para antibióticos macrólidos. Por lo tanto, la inhibición de tales expresión de la molécula de adhesión podría explicar, al menos en parte, el mecanismo de macrólidos inducida atenuación de la acumulación de neutrófilos en las vías respiratorias.

\section{Efectos sobre las células epiteliales}

La actividad de los macrólidos, y su relación con las células epiteliales tiene un papel importante en el efecto inmunomodulador a través de la supresión de citokinas y factores de adherencia como se ha demostrado en diferentes ensayos. 
La azitromicina reduce significativamente la secreción de TNFa por IB3-1 las células de las vías respiratorias con $F Q$, a niveles comparables a los de CFTR-corregida isogénicas C38 línea celular (31). Azitromicina también inhibe la producción de IL-8, y NFKB y AP-1 en las actividades de carácter vinculante las células epiteliales de pulmón (32). Eritromicina y sus derivados disminuir la actividad de NF $\kappa B$ y AP-1 en el ser humano de células bronquiales BET línea $1 \mathrm{~A}$, lo que demuestra que la actividad inmunomoduladora y antimicrobiana de los macrólidos se puede separar (33). Macrólidos suprimen la liberación de los neutrófilos epiteliales derivadas de factores de supervivencia, incluido el GM-CSF (34). Curiosamente, sin efectos directos de los macrólidos en la apoptosis de los neutrófilos y la supervivencia se observaron en este estudio (34).

\section{Efecto sobre la defensa del epitelio}

Daños en el epitelio es una característica importante en el asma, EPOC, fibrosis quística y otras enfermedades pulmonares inflamatorias. El mantenimiento de la integridad y la función de la barrera epitelial es fundamental para prevenir la remodelación de pulmón en el asma y la $\operatorname{EPOC}(35,36)$. Formando una barrera física y química, las células epiteliales y secreciones mucosas proporcionan una primera línea de defensa contra agentes patógenos y sustancias irritantes en el pulmón. Esta barrera epitelial anatómica e inmunológica, que incluye la creación de complejos de unión y la determinación de la polaridad de las células epiteliales y la producción de moco, sustancias antimicrobianas y tensioactivos. Un programa de respuestas del epitelio coordinadas, incluida la migración, la proliferación, y el montaje de la matriz, se establece rápidamente después de una lesión para restaurar la integridad de los tejidos dañados. La plasticidad y la capacidad de re-diferenciación de las células epiteliales de pulmón son componentes críticos de este mecanismo de reparación. Las células epiteliales también dirigen la respuesta inflamatoria en las vías respiratorias y modulan el reclutamiento de células inflamatorias, a través de la producción de citoquinas y quimiquinas y la modulación de la expresión de receptores y moléculas de adhesión. Por último, las células epiteliales están implicados en la liquidación de los microorganismos de las vías respiratorias, a través de mecanismos que implican el reclutamiento de neutrófilos, la producción de sustancias antimicrobianas, la apoptosis de las células epiteliales infectadas y la depuración mucociliar .

\section{Efectos en el moco}

El moco es la primera barrera de defensa de las vías respiratorias y está compuesto de agua, proteínas de mucina, los fosfolípidos del surfactante y proteínas y péptidos de defensa secretada por las células epiteliales. En muchas enfermedades pulmonares, como asma, EPOC y fibrosis quística, los cambios en el resultado del epitelio en la composición de la secreción anormal, alteración del moco o propiedades de alteración en la depuración, ponen en peligro la depuración mucociliar y favorecen la infección y la inflamación crónica (37). Los macrólidos pueden modular la expresión de genes de mucina y la producción de proteínas. Claritromicina inhibe la hiperplasia de células caliciformes y metaplasia y la infiltración de neutrófilos (38).Claritromicina inhibe TNFa inducida por la secreción de mucina, mientras que la eritromicina inhibe la secreción de mucina sólo basal, josamicina, no tuvo ningún efecto (38). Claritromicina inhibe la producción de proteínas MUC5AC en un modelo de ratón de la infección por P. aeruginosa, la señalización en este modelo depende de la activación de ERK (39).

Las proteínas secretadas por las bacterias pueden inducir la secreción de mucina epitelial. Azitromicina inhibe la producción de MUC5AC inducida por P. aeruginosa sensible mediador en la línea humana de células epiteliales (40). Cabe destacar que la $P$. aeruginosa se considera resistente a la acción puramente a los antibióticos de los macrólidos. El mecanismo de regulación MUC5AC es a través de la activación de ERK y vías NFKB (40), en consonancia con el efecto de los macrólidos en las vías de señalización en las células epiteliales de la vía aérea principal. Aunque los estudios en las vías respiratorias se han centrado en MUC5AC principal de la mucina producida por las células caliciformes de superficie, el efecto de los macrólidos en la expresión de genes de mucina y / o la producción de proteínas se puede extender a otras mucinas y superficies epiteliales, por ejemplo, roxitromicina suprime la expresión génica MUC2 en cultivos de células epiteliales intestinales, el efecto consiste en la inhibición de la NFKB de señalización, como se indica en los estudios de análisis de periodista (42). En general, el efecto inhibitorio de los macrólidos en hipersecreción mucosa puede explicarse por su actividad inmunomoduladora, así como los efectos inhibidores directos de la actividad enzimática de la elastasa de los neutrófilos (43).

\section{Efectos en el movimiento de iones}

El Movimiento de los iones a través del epitelio de las vías respiratorias regula la hidratación y la altura de la capa de líquido periciliar; una altura igual a la altura completamente extendida de un cilio parece ser óptimo para la propulsión de la capa de moco. El regulador de la conductancia transmembrana de la FQ (CFTR, un canal de cloruro) y el canal de sodio epitelial (ENAC) 
son canales iónicos que regulan en la vía aérea el transporte de iones y agua. Volumen de líquido de la superficie de la vía aérea se rige por fuerzas osmóticas a través del epitelio de las vías respiratorias incluyendo la absorción de sodio y la secreción de cloro, y el movimiento del agua tras el flujo de iones La Disregulación de transporte iónico epitelial se asocia con disfunción eliminación del muco, en la FQ y la EPOC. Aunque los estudios pilotos la mejoría en la conductancia de cloruro de un tratamiento corto con azitromicina en la fibrosis quística (44), y la modulación del transporte de cloro por la eritromicina (que podría resultar en la secreción de moco reducido) en cultivos de células epiteliales bronquiales (BEAS-2B) (45), más amplios estudios muestran que la claritromicina y azitromicina no tienen ningún efecto sobre el transporte de iones en cualquiera de los modelos de ratón o de seres humanos con FQ (46). Los datos experimentales y clínicos indican que los efectos beneficiosos de los macrólidos en la FQ no es a causa de la mejora del transporte de iones epiteliales (47) continua siendo un misterio la forma como los macrolidos regulan la secreción de moco y el movimiento ciliar. Nuevos estudios deberán dilucidar este interrogante.

\section{Efecto sobre las defensinas}

Las defensinas están ampliamente distribuidos en péptidos antimicrobianos endógenos. En particular, epiteliales $\beta$-defensinas son un componente importante de la inmunidad innata en las vías respiratorias (47, 48). La eritromicina aumenta $\beta$-defensina actividad antimicrobiana depende de líquido de superficie de los cultivos humanos de células epiteliales primarias de la tráquea y las líneas celulares humanas, incluyendo BEAS-2B bronquiales y alveolares de tipo II-como las células A549 [49•]. In vivo, los macrólidos se han mostrado una disminución de $\beta$-defensina- 2 niveles en el líquido broncoalveolar de pacientes con DPB, probablemente como consecuencia de la supresión de la inflamación (49).

\section{EFECTOS SOBRE LA ESTABILIDAD DEL EPITELIO}

Se plantea la posibilidad de que los macrólidos pueden contribuir al mantenimiento de la barrera epitelial física por la estabilización de los complejos de unión. Esto principalmente con la eritromicina. La roxitromicina ha mostrado efectos citoprotectoras en humanos, células primarias de las vías aéreas bronquiales y pequeñas (50).

Estas observaciones sobre el efecto de los macrólidos en la protección del epitelio puede explicar en parte los efectos beneficiosos de estos fármacos en pacientes con enfermedades pulmonares crónicas. Los macrólidos puede modular vías diferentes, que participan en la integridad epitelial, la defensa y, posiblemente, de reparación, dependiendo de la clase de los macrólidos y la activación de los estímulos (17).

\section{CONCLUSION}

En las enfermedades respiratorias inflamatorias, donde el papel de los neutrofilos, moléculas de adhesión, citoquinas, y mediadores de respuesta inflamatoria tienen un papel preponderante, surge un grupo de medicamentos, utilizados por años su efecto bactericida como una nueva herramienta en el arsenal terapéutico, pero no por su efecto conocido, ya se ha documentado in vitro un efecto inmunomodulador, inhibiendo la producción de IL8, de moléculas de adhesión como ICAM1, por mecanismos de movimiento ionico, los cuales tienen un papel fundamental en el barrido mucociliar, en la regulación y calidad de producción de moco a través de factores de necrosis, adicionalmente del efecto antibiótico. Gracias a estos efectos los macrolidos pueden tener una papel mayor del reconocido hasta hoy, en patológicas como la Panbronquiolitis difusa, Fibrosis Quistica, Asma, Enfermedad Pulmonar Obstructiva Crónica.

Sin embargo estas revisiones se basan en pocos estudios, por lo cual la invitación es para la realización de nuevos estudios aleatorizados, con grupo control en el cual podamos establecer por medio de la evidencia el papel de los macrolidos en este tipo de patologías. No hay que desconocer el riesgo de inducir resistencia, sin embargo surge hoy una alternativa para el manejo del EPOC, que pudiera disminuir las recidivas, mejorar el VEF1 y la calidad de vida de nuestros pacientes.

\section{REFERENCIAS}

1. Comité Científico del Estudio IBERPOC. Proyecto IBERPOC: un estudio epidemiológico de la EPOC en España. Arch Bronconeu - mol. 1997;33:293-9.

2. Manfreda J, Mas Y, Litven W. Morbidity and mortality from chronic obstructive pulmonary disease. Am Rev Respir Dis.1989;140:19-26.

3. Hurd S. The impact of COPD on lung health worldwide. Epidemiology and incidence. Chest. 2000;117:1-4.

4. Tateda, T.J. Standiford, J.C. Pechere and K. Yamaguchi, Regulatory effects of macrolides on bacterial virulence: potential role as quorum-sensing inhibitors, Curr Pharm Des 10 (2004), pp. 3055-3065

5. B.K. Rubin and M.O. Henke, Immunomodulatory activity and effectiveness of macrolides in chronic airway disease, Chest 125 (2004), pp. 70S-78S.

6. M. Shinkai and B.K. Rubin, Macrolides and airway inflammation in children, PaediatrRespir Rev 6 (2005), pp. 227-235. 
7. M.O. Henke, S.A. Shah and B.K. Rubin, The role of airway secretions in COPD - clinical applications, COPD 2 (2005), pp. 377-390

8. B.K. Rubin and M.O. Henke, Immunomodulatory activity and effectiveness of macrolides in chronic airway disease, Chest 125 (2004), pp. 70S-78S.

9. S.L. Johnston, Macrolide antibiotics and asthma treatment, J Allergy Clinlmmunol 117 (2006), pp. 1233-1236.

10. M.J. Parnham, Immunomodulatory effects of antimicrobials in the therapy of respiratory tract infections, CurrOpin Infect Dis 18 (2005), pp. 125-131.

11. Giamarellos-Bourboulis EJ: Macrolides beyond the conventional antimicrobials: a class of potent immunomodulators. Int J Antimicrob Agents2008, 31:12-20.

12. M. Shinkai, G.H. Foster and B.K. Rubin, Macrolide antibiotics modulate ERK phosphorylation and IL-8 and GM-CSF production by human bronchial epithelial cells, Am J Physiol Lung Cell MolPhysiol 290 (2006), pp. L75-L85.

13. M. Shinkai, Y.S. Lopez-Boado and B.K. Rubin, Clarithromycin has an immunomodulatory effect on ERK-mediated inflammation induced by Pseudomonas aeruginosaflagellin, J AntimicrobChemother 59 (2007), pp. 1096-1101.

This was one of the first papers demonstrating that the effect of low dose macrolides is truly immunomodulatory rather than anti-inflammatory, and that this was mediated, in part, by the ERK pathway, confirming earlier work (reference [10]).

14. M. Shinkai, J. Tamaoki, H. Kobayashi, S. Kanoh, K. Motoyoshi, T. Kute and B.K. Rubin, Clarithromycin delays progression of bronchial epithelial cells from $\mathrm{G} 1$ phase to $\mathrm{S}$ phase and delays cell growth via extracellular signal-regulated protein kinase suppression, Antimicrob Agents Chemother 50(2006), pp. 17381744.

15. L. Saiman, B.C. Marshall, N. Mayer-Hamblett, J.L. Burns, A.L. Quittner, D.A. Cibene, S. Coquillette, A.Y. Fieberg, F.J. Accurso and P.W. Campbell III, Azithromycin in patients with cystic fibrosis chronically infected with Pseudomonas aeruginosa: a randomized controlled trial, JAMA 290 (2003), pp. 1749-1756.

16. M.J. Schultz, Macrolide activities beyond their antimicrobial effects: macrolides in diffuse panbronchiolitis and cystic fibrosis, J AntimicrobChemother. (2004), pp. 21-28.

17. W.C. Tsai and T.J. Standiford, Immunomodulatory effects of macrolides in the lung: lessons from in-vitro and invivo models, Curr Pharm Des 10(2004), pp. 3081-3093.

18. Shinkai M, HenkeMO, Rubin BK: Macrolide antibiotics as immunomodulatory medications: proposed mechanisms of action. Pharm Therap2008, 117:393-405.

19. O. Culic, V. Erakovic, I. Cepelak, K. Barisic, K. Brajsa, Z. Ferencic, R. Galovic, I. Glojnaric, Z. Manojlovic and V. Munic et al., Azithromycin modulates neutrophil function and circulating inflammatory mediators in healthy human subjects, Eur $\mathrm{J}$ Pharmacol 450 (2002), pp. 277-289.

20. M.J. Parnham, O. Culic, V. Erakovic, V. Munic, S. Popovic-Grle, K. Barisic, M. Bosnar, K. Brajsa, I. Cepelak and S. Cuzic et al., Modulation of neutrophil and inflammation markers in chronic obstructive pulmonary disease by short-term azithromycin treatment, Eur J Pharmacol 517 (2005), pp. 132-143

This study shows that in vivo, azithromycin can initially increase the elaboration of inflammatory mediators followed by a sustained decrease in inflammation, as is characteristic of immunomodulation.
21. T.V. Ivetic, B. Bosnjak, B. Hrvacic, M. Bosnar, N. Marjanovic, Z. Ferencic, K. Situm, O. Culic, M.J. Parnham and V. Erakovic, Antiinflammatory activity of azithromycin attenuates the effects of lipopolysaccharide administration in mice, Eur $J$ Pharmacol 539 (2006), pp. 131-138.

22 E.J. Giamarellos-Bourboulis, T. Adamis, G. Laoutaris, L. Sabracos, V. Koussoulas, M. Mouktaroudi, D. Perrea, P.E. Karayannacos and $\mathrm{H}$. Giamarellou, Immunomodulatory clarithromycin treatment of experimental sepsis and acute pyelonephritis caused by multidrug-resistantPseudomonasaeruginosa, Antimicrob Agents Chemother 48 (2004), pp. 93-99.

23. R. Legssyer, F. Huaux, J. Lebacq, M. Delos, E. Marbaix, P. Lebecque, D. Lison, B.J. Scholte, P. Wallemacq and T. Leal, Azithromycin reduces spontaneous and induced inflammation in DeltaF508 cystic fibrosis mice, Respir Res 7 (2006), p. 134. It is thought that CF lung disease is due, in part, to a hyperinflammatory state, with both constitutive inflammation and the induced inflammatory response being increased. This study demonstrates that azithromycin can 'reset' (modulate) both the spontaneous and the induced inflammatory response in a mouse model of CF.

M. Bosnar, Z. Kelneric, V. Munic, V. Erakovic and M.J. Parnham, Cellular uptake and efflux of azithromycin, erythromycin, clarithromycin, telithromycin, and cethromycin, Antimicrob Agents Chemother 49 (2005), pp. 2372-2377.

24. M. Bosnar, Z. Kelneric, V. Munic, V. Erakovic and M.J. Parnham, Cellular uptake and efflux of azithromycin, erythromycin, clarithromycin, telithromycin, and cethromycin, Antimicrob Agents Chemother 49 (2005), pp. 2372-2377.

25. S. Hodge, G. Hodge, S. Brozyna, H. Jersmann, M. Holmes and P.N. Reynolds, Azithromycin increases phagocytosis of apoptotic bronchial epithelial cells by alveolar macrophages, EurRespir J 28 (2006), pp. 486-495.

26. T. Yamaryo, K. Oishi, H. Yoshimine, Y. Tsuchihashi, K. Matsushima and T. Nagatake, Fourteen-member macrolides promote the phosphatidylserine receptor-dependent phagocytosis of apoptotic neutrophils by alveolar macrophages, Antimicrob Agents Chemother 47 (2003), pp. 48-53These two interesting papers postulate a new effect of macrolides in resolution of lung inflammation. Macrolides can improve phagocytosis of apoptotic cells by alveolar macrophages, a critical component of resolution of chronic inflammation (refs. [

27. S. Hodge, G. Hodge, R. Scicchitano, P.N. Reynolds and M. Holmes, Alveolar macrophages from subjects with chronic obstructive pulmonary disease are deficient in their ability to phagocytose apoptotic airway epithelial cells, Immunol Cell Biol 81 (2003), pp. 289-296

28. S. Hodge, G. Hodge, J. Ahern, H. Jersmann, M. Holmes and P.N. Reynolds, Smoking alters alveolar macrophage recognition and phagocytic ability: implications in COPD, Am J Respir Cell MolBiol 37 (2007), pp. 748-755

29. Y.J. Li, A. Azuma, J. Usuki, S. Abe, K. Matsuda, T. Sunazuka, T. Shimizu, Y. Hirata, H. Inagaki and T. Kawada et al., EM703 improves bleomycin-induced pulmonary fibrosis in mice by the inhibition of TGF-beta signaling in lung fibroblasts, Respir Res 7 (2006), p. 16. This study demonstrates that a powerfully immunomodulatory 14-member macrolide derivative can actually hasten resolution of bleomycin-induced lipid peroxidation injury and fibrosis. This is also a remarkable paper because EM703 is a derivative of erythromycin $A$ that has no identifiable antimicrobial properties.

30. Ikeda H, Sunazuka T, Suzuki H, Hamasaki Y, Yamazaki S, Omura $S$, Hatamochi A: EM703, the new derivative of erythromycin, 
inhibits transcription of type I collagen in normal and scleroderma fibroblasts. J DermatolSci 2008, 49:195-205

31 C. Cigana, B.M. Assael and P. Melotti, Azithromycin selectively reduces tumor necrosis factor alpha levels in cystic fibrosis airway epithelial cells,Antimicrob Agents Chemother 51 (2007), pp. 975-981.

32. C. Cigana, E. Nicolis, M. Pasetto, B.M. Assael and P. Melotti, Antiinflammatory effects of azithromycin in cystic fibrosis airway epithelial cells,BiochemBiophys Res Commun 350 (2006), pp. 977-982

33. M. Desaki, H. Okazaki, T. Sunazuka, S. Omura, K. Yamamoto and $\mathrm{H}$. Takizawa, Molecular mechanisms of anti-inflammatory action of erythromycin in human bronchial epithelial cells: possible role in the signaling pathway that regulates nuclear factor-kappaB activation, Antimicrob Agents Chemother 48 (2004), pp. 1581-1585

This paper illustrates the molecular mechanisms of macrolide activity in a lung epithelial cells line, including the non-antimicrobial erythromycin derivative EM703.

34. H. Yamasawa, K. Oshikawa, S. Ohno and Y. Sugiyama, Macrolides inhibit epithelial cell-mediated neutrophil survival by modulating granulocyte macrophage colony-stimulating factor release, Am J Respir Cell MolBiol 30 (2004), pp. 569-575.

35. H. Takizawa, M. Desaki, T. Ohtoshi, S. Kawasaki, T. Kohyama, M. Sato, M. Tanaka, T. Kasama, K. Kobayashi and J. Nakajima et al., Erythromycin modulates IL-8 expression in normal and inflamed human bronchial epithelial cells, Am J RespirCrit Care Med 156 (1997), pp. 266-271.

36. T. Sunazuka, H. Takizawa, M. Desaki, K. Suzuki, R. Obata, K. Otoguro and S. Omura, Effects of erythromycin and its derivatives on interleukin-8 release by human bronchial epithelial cell line BEAS-2B cells, J Antibiot (Tokyo) 52 (1999), pp. 7174.

36. S.H. Randell and R.C. Boucher, Effective mucus clearance is essential for respiratory health, Am J Respir Cell MolBiol 35 (2006), pp. 20-28.

This is an excellent recent review on mucociliary clearance and its critical role for normal lung function.

37. T. Shimizu, S. Shimizu, R. Hattori, E.C. Gabazza and Y. Majima, In vivo and in vitro effects of macrolide antibiotics on mucus secretion in airway epithelial cells, Am J RespirCrit Care Med 168 (2003), pp. 581-587. 38. Y. Kaneko, K. Yanagihara, M. Seki, M. Kuroki, Y. Miyazaki, Y. Hirakata, H. Mukae, K. Tomono, J. Kadota and S. Kohno, Clarithromycin inhibits overproduction of muc5ac core protein in murine model of diffuse panbronchiolitis, Am J Physiol Lung Cell MolPhysiol 285 (2003), pp. L847-L853.

39. V. Sperandio, Novel approaches to bacterial infection therapy by interfering with bacteria-to-bacteria signaling, Expert Rev Anti Infect Ther 5 (2007), pp. 271-276

40. W.E. Swords and B.K. Rubin, Macrolide antibiotics, bacterial populations and inflammatory airway disease, Neth $\mathrm{J}$ Med 61 (2003), pp. 242-248.

41. Y. Imamura, K. Yanagihara, Y. Mizuta, M. Seki, H. Ohno, Y. Higashiyama, Y. Miyazaki, K. Tsukamoto, Y. Hirakata and K. Tomono et al., Azithromycin inhibits MUC5AC production induced by the Pseudomonas aeruginosaautoinducer $\mathrm{N}$-(3Oxododecanoyl) homoserine lactone in NCl-H292 Cells, Antimicrob Agents Chemother 48 (2004), pp. 3457-3461.

42. D.Y. Kim, K. Takeuchi, H. Ishinaga, C. Kishioka, S. Suzuki, C. Basbaum and Y. Majima, Roxithromycin suppresses mucin gene expression in epithelial cells, Pharmacology 72 (2004), pp. 6-11.

43. U. Pradal, A. Delmarco, M. Morganti, M. Cipolli, E. Mini and G. Cazzola, Long-term azithromycin in cystic fibrosis: another possible mechanism of action?, J Chemother 17 (2005), pp. 393400 .

44. N. Ge, Y. Nakamura, Y. Nakaya and S. Sone, Interferon-gamma activates outwardly rectifying chloride channels in the human bronchial epithelial cell line BEAS-2B, J Med Invest 48 (2001), pp. 97-101.

45. P.M. Barker, D.J. Gillie, M.S. Schechter and B.K. Rubin, Effect of macrolides on in vivo ion transport across cystic fibrosis nasal epithelium, Am J RespirCrit Care Med 171 (2005), pp. 868-871

46. D.M. Laube, S. Yim, L.K. Ryan, K.O. Kisich and G. Diamond, Antimicrobial peptides in the airway, Curr Top Microbiollmmunol 306 (2006), pp. 153-182.

47. K. Ishizawa, T. Suzuki, M. Yamaya, Y.X. Jia, S. Kobayashi, S Ida, H. Kubo, K. Sekizawa and H. Sasaki, Erythromycin increases bactericidal activity of surface liquid in human airway epithelial cells, Am J Physiol Lung Cell MolPhysiol 289 (2005), pp. L565L573.

48. This report shows that macrolides can increase epithelial antimicrobial activity in the absence of inflammation, suggesting that macrolide treatment may contribute to improvement of the epithelial barrier function and defense against microorganisms by this mechanism.

49. T. Hiratsuka, H. Mukae, H. liboshi, J. Ashitani, K. Nabeshima, T. Minematsu, N. Chino, T. Ihi, S. Kohno and M. Nakazato, Increased concentrations of human beta-defensins in plasma and bronchoalveolar lavage fluid of patients with diffuse panbronchiolitis, Thorax 58 (2003), pp. 425-430

50. X. Gao, R. Ray, Y. Xiao, P.E. Barker and P. Ray, Inhibition of sulfur mustard-induced cytotoxicity and inflammation by the macrolide antibiotic roxithromycin in human respiratory epithelial cells, BMC Cell Biol 8 (2007), p. 17

51. Timothy R. Pasquale and James S. Tan, Nonantimicrobial effects of antibacterial agents, clinical infectious diseases 2005;40;127135.

52. Jun Tamaoki, The effects of Macrolides on Inflammatory Cells, CHEST,2004;125;41s-51s.

53. Esterly NB, Furey NL, Flanagan LE. The effect of antimicrobial agents on leukocyte chemotaxis. J Invest Dermatol 1978; 70:51-55.

54. Nelson S, Summer WR, Terry PB, et al. Erythromycininduced suppression of pulmonary antibacterial defenses: a potential mechanism of superinfection in the lung. Am Rev Respir Dis 1987; 136:1207-1212.

55. Kawasaki S, Takizawa H, Takayuki O, et al. Roxithromycin inhibits cytokine production by and neutrophil attachment to human bronchial epithelial cells in vitro. Antimicrob Agents Chemother 1998; 42:1499-1502. 